\title{
Analysis of the Intestinal Microbiome of a Recovered Clostridium difficile Patient after Fecal Transplantation
}

\author{
Felix Broecker ${ }^{a, d, g} \quad$ Michael Kube $^{e}$ Jochen Klumpp ${ }^{b}$ Markus Schuppler ${ }^{b}$ \\ Luc Biedermann ${ }^{c}$ Jochen Hecht ${ }^{f}$ Michael Hombach ${ }^{a}$ Peter M. Keller ${ }^{a}, \mathrm{~h}$ \\ Gerhard Rogler ${ }^{c}$ Karin Moelling ${ }^{a, d, i}$ \\ anstitute of Medical Microbiology, University of Zurich, ${ }^{b}$ Institute of Food, Nutrition and Health, ETH Zurich, \\ 'Division of Gastroenterology and Hepatology, University Hospital Zurich, Zurich, Switzerland, dMax Planck \\ Institute for Molecular Genetics, 'Department of Crop and Animal Sciences, Humboldt-Universität zu Berlin, \\ ${ }^{f}$ Berlin-Brandenburg Center for Regenerative Therapies, Berlin, 9 Max Planck Institute of Colloids and Interfaces, \\ Potsdam, h Department of Internal Medicine IV, Gastroenterology, Hepatology and Infectious Diseases, University \\ Hospital Jena, Jena, and 'Heinrich Pette Institute, Hamburg, Germany
}

\section{Key Words}

Clostridium difficile - Clostridium difficile-associated disease . Colitis - Fecal microbiota transplantation - Metagenomics . Microbiome $\cdot$ Illumina

\footnotetext{
Abstract

Background: Clostridium difficile infections upon antibiotic disruption of the gut microbiota are potentially lethal. Fecal microbiota transplantation (FMT) is a promising treatment option for recurrent $C$. difficile-associated disease (CDAD). Here, we present a patient with recurrent CDAD that received FMT, leading to full recovery for what has now been 3 years. We performed metagenomic sequencing on stool samples to assess if there are indications for recolonization with C. difficile and changes in the gut microbiota after FMT. Methods: DNA from the stool of the donor and recipient was subjected to illumina sequencing. Obtained read sets were assembled to contiguous sequences and open reading frames were predicted. Deduced proteins were taxonomically assigned. Results: We detected complex and apparently healthy microbiomes in the donor's and recipient's intes-
}

tines after FMT, but no indications for $C$. difficile colonization. Conclusions: Metagenomic analysis proved suitable to analyze the intestinal microbiome after FMT. Discussion of our evaluation procedure and data management may be helpful for future studies. We demonstrated restoration of a healthy and diverse gut microbiome with chimeric composition from donor and recipient, and long-lasting clearance of C. difficile. The procedure is simple, cheap, caused no side effects, and was stable over 3 years.

ㄷ) 2013 S. Karger AG, Basel

\section{Introduction}

Hospital-acquired infections with Clostridium difficile are the most common cause of antibiotic-associated diarrhea [1]. Antibiotic disruption of the intestinal microbiota followed by colonization with toxin-producing C. difficile spores commonly found in healthcare facilities causes $C$. difficile-associated disease (CDAD), which in severe cases requires colectomy or leads to death [1]. The emergence of new $C$. difficile strains, such as ribo-

\section{KARGER}

E-Mail karger@karger.com www.karger.com/dig
(C) 2013 S. Karger AG, Basel

0012-2823/13/0884-0243\$38.00/0
Prof. Dr. Karin Moelling

Institute of Medical Microbiology, University of Zurich Gloriastrasse 30/32

$\mathrm{CH}-8006$ Zurich (Switzerland)

E-Mail moelling@imm.uzh.ch 
type 027 (also designated NAP1, BI or toxin type III) with increased virulence, toxin expression and antibiotic resistance may be responsible for an increase in CDAD incidence and death rates during the last years $[2,3]$. In addition to the elderly as the main risk group, children, young adults and pregnant women are increasingly becoming infected [4-6]. Ribotype 027 has quickly spread throughout America, Europe, Asia and Oceania [7].

Current treatment relies on the antibiotics metronidazole or vancomycin [8-10]. However, despite efficient antibiotic treatment of individual episodes, about $30 \%$ of patients suffer from one or more recurrent CDAD episodes [11-13], which has led to the investigation of fecal bacteriotherapy, or fecal microbiota transplantation (FMT), as an alternative treatment option [14-37].

The objective of FMT is restoring an intact normal intestinal microbiota, which prevents overgrowth by pathogenic $C$. difficile. Donor stool for this kind of treatment is usually obtained from a spouse/intimate partner or relative tested for various pathogens prior to transplantation. Anecdotal reports and various smaller case series have suggested a high success rate $(80-100 \%)$ of FMT to treat recurrent CDAD [14-36]. Moreover, the first controlled randomized clinical trial has recently reported the superiority of FMT to standard antibiotic therapy [37]. FMT has not been widely implemented in clinical practice until today, likely due to safety concerns and the risk of transmitting infectious pathogens from donor to recipient $[22,25,27]$.

Successful treatment of CDAD by FMT depends on establishing a normal microbiome in the recipient's intestinal tract while avoiding transmission of pathogenic microorganisms. This requires a reliable means of surveillance of the microbiome in the recipient. To address this issue, we investigated a metagenomics approach using sequencing-by-synthesis (illumina sequencing) to obtain insights into the microbiome of a CDAD patient who underwent successful FMT after antibiotic therapy failed repeatedly. Upon FMT, the patient fully recovered and has shown no signs of CDAD for what has now been 3 years since FMT.

To detect a potential persistent CDAD infection, we analyzed the composition of the intestinal microbiome to follow the treatment course over time through metagenomic deep sequencing. Our approach allowed detection of intestinal microorganisms up to the species level. We found no evidence for the presence of a C. difficile infection in the recipient's intestinal microbiome, providing scientific evidence not only for the clinical efficacy of FMT, but also of its potential to successfully eliminate potential pathogenic residues of $C$. difficile in this patient.

\section{Case History}

A 51-year-old woman was admitted to our hospital with her sixth episode of recurrent CDAD. The patient was afebrile and denied weight loss or hematochezia, but suffered from severe diarrhea (6-8 times per day) that had reoccurred 2 weeks after cessation of oral vancomycin. On admission, the patient presented with normal vital signs. The abdominal exam revealed a mildly and diffusely tender abdomen without involuntary guarding or rebound tenderness.

Stool samples of this patient tested negative by standard diagnostic tests for Salmonella, Shigella, Campylobacter, Vibrio, Aeromonas and Plesiomonas spp., enterovirulent Escherichia coli, and intestinal parasites. However, the VIDAS C. difficile toxin A and B immune assay (EIA; BioMérieux, Marcy L'Étoile, France) was positive. Selective cultures on cycloserine-cefoxitin-fructose agar (BD Difco, Franklin Lakes, N.J., USA) followed by confirmation of suspect colonies with an agglutination assay (Microgen C. difficile; Microgen Bioproducts Ltd., Camberley, UK) grew C. difficile. A PCR assay for C. difficile toxin genes $t c d A$ and $t c d B$ (Xpert C. difficile; Cepheid, Sunnyvale, Calif., USA) was positive as well. For antimicrobial susceptibility testing, minimal inhibitory concentrations of metronidazole and vancomycin were determined with the Etest assay (BioMérieux, Marcy L'Étoile, France). Minimal inhibitory concentration for rifampicin (Sigma-Aldrich $\mathrm{GmbH}, \mathrm{Zug}$, Switzerland) as a surrogate for rifaximin was determined using the microdilution method according to the guidelines of the Clinical Laboratory Standards Institute (CLSI) [38]. Minimal inhibitory concentrations were in the susceptible range with $0.5 \mathrm{mg} / \mathrm{l}$ (vancomycin), $0.25 \mathrm{mg} / \mathrm{l}$ (metronidazole) and $<0.002$ $\mathrm{mg} / \mathrm{l}$ (rifampicin).

The patient's personal medical history comprised recurrent migraine headaches (currently around 2 episodes per month), perforated appendicitis with peritonitis (around 30 years beforehand), repetitive laparoscopic investigations with regard to endometriosis, and cystic lesions of the ovary and hemithyroidectomy due to unilateral toxic goiter (requiring thyroid consecutive hormone replacement).

Two years before the current admission, the patient suffered from a complicating jawbone infection requiring 3 surgical debridements. Peroral (p.o.) antibiotic therapy was installed for a total of 7 months and the antibiotic regimen was changed several times due to insufficient clinical response. Antibiotic therapy was started with clindamycin, metronidazole combined with amoxicillin/clavulanic acid and amoxicillin/clavulanic acid alone. Infection could finally be cured after 10 weeks of amoxicillin/clavulanic acid. In the course of the longstanding antibiotic treatment, the patient suffered from the first CDAD episode with severe diarrhea 9 months before the current hospital admission.

As an initial treatment, according to the current recommendations [10], metronidazole $500 \mathrm{mg}$ p.o. 3 times a day for 7 days in conjunction with the probiotic Saccharomyces cerevisiae (commonly referred to as $S$. boulardii) was selected. Symptoms promptly resolved upon antibiotic therapy. Four days after the end of treatment, the first recurrent CDAD episode occurred, which was successfully treated with antibiotic therapy of $125 \mathrm{mg}$ vancomycin p.o. 4 times a day (q.i.d.) for 14 days. However, a second recurrence occurred 1 month after cessation of vancomycin. Treatment with vancomycin was started again with a decreasing dosing scheme of $750 \mathrm{mg}$ p.o. per day, $500 \mathrm{mg}$ p.o. per 
day and $375 \mathrm{mg}$ p.o. per day for 1 week each followed by $125 \mathrm{mg}$ p.o. q.i.d. every other day for 2 weeks and $125 \mathrm{mg}$ p.o. q.i.d. every third day for another 3 weeks, as recommended [10]. Two weeks after cessation of antibiotic therapy, a third CDAD episode occurred. Again, therapy with vancomycin was initiated with an increased initial daily dose of $500 \mathrm{mg}$ p.o. q.i.d. which was subsequently decreased to $250 \mathrm{mg}$ q.i.d. [10]. During the treatment course, a vaginal $S$. cerevisiae infection occurred, potentially resulting from the previous use of the prebiotic containing this particular yeast. This infection was successfully treated with fluconazole. After a total of 6 weeks of vancomycin, the antibiotic regimen was changed to rifaximin $400 \mathrm{mg}$ p.o. 2 times a day for 14 days due to persisting symptoms [39]. Eight weeks after cessation of rifaximin therapy, a fourth CDAD episode emerged. A therapeutic trial with intravenous polyclonal immunoglobulins (Privigen ${ }^{\circledR}$ ) 20 g given intravenously once was conducted without a significant clinical response. At this time, colonoscopy revealed no obvious abnormalities of the colonic and ileal mucosae, although an abnormal friability was observed.

As standard treatment options remained unsuccessful, rescue treatment options were discussed between the clinicians and microbiologists. The recently published approach of a targeted therapy with a monoclonal antibody against $C$. difficile toxins [40] was evaluated, but could not be performed as this drug was not yet available for clinical use.

After discussion with the patient, FMT (also referred to as fecal bacteriotherapy) was performed with donor feces from the patient's sister. Fresh donor feces (10 min after defecation) were prepared by (1) resuspension of a cherry-sized amount of feces in $120 \mathrm{ml}$ of sterile $0.9 \% \mathrm{NaCl}$ solution (B. Braun Melsungen AG, Melsungen, Germany), (2) two filtration steps through sterile gauze and (3) transferring $50 \mathrm{ml}$ of the resulting bacterial suspension in each of two Entocort bottles (AstraZeneca) by intra-anal application. All preparation steps were performed in a laminar flow cabinet under biosafety level 2 conditions. Over the last 2 months prior to FMT, the patient took vancomycin $125 \mathrm{mg}$ q.i.d. p.o. The donor tested negative for HIV, HTLV, and hepatitis B and $\mathrm{C}$ infections, as well as negative for the presence of C. difficile, Salmonella, Shigella, Campylobacter, Yersinia, Vibrio, Aeromonas and Plesiomonas spp., and enteropathogenic E. coli. The FMT procedure was conducted within $30 \mathrm{~min}$ of preparation of the donor suspension by parallel application of the two bacteriotherapeutic enemas. The patient subsequently reported changes in the frequency of bowel movements and intermittent obstipation, both of which ceased after 10 weeks. To date, the patient remains free of any symptoms (which has been roughly 3 years since FMT) and has reported significant amelioration of her general physical condition. After FMT, the patient underwent two short antibiotic treatments, which did not cause any side effects.

\section{Results}

\section{Metagenomic Analysis of Intestinal Microbiome after FMT}

FMT is a promising alternative to antibiotic treatment for recurrent $\mathrm{CDAD}$ [14-37]. However, the nature of successful FMT with respect to the microbial diversity in the recipient remains largely unknown. In accordance with our results, two recent studies have reported an increase in complexity and richness of patients' intestinal microbiomes after FMT, employing 16S rRNA deep sequencing of recipients' stool samples [21,29]. Metagenome analysis by deep sequencing is a powerful tool to assess the microbial diversity of the gut [41], and has previously been widely applied to assess the composition and changes of the intestinal microbiota in health and disease, and as a consequence of environmental factors [42]. To study the diversity of the intestinal microbiome of the patient presented here over time, we performed metagenomic deep sequencing of stool samples obtained at different time points from the recipient (the patient) and donor (the patient's sister).

To this end, we analyzed the donor stool sample at the time of FMT (donor, April 4, 2010; D0), as well as stool samples of the recipient 6-7 months after FMT in 2-week intervals (recipient, November 1, 2010, November 12, 2010 and November 11, 2010; R1, R2 and R3). The recipient's sample at the time of FMT was unavailable due to inappropriate storage. DNA was isolated from about $200 \mathrm{mg}$ of frozen feces with the QIAamp DNA Stool Mini Kit (Qiagen), treated with DNase-free RNase (Fermentas) and further purified by phenol-chloroform extraction. DNA was subjected to the illumina sequencing-bysynthesis approach. Barcoded libraries were generated with 3-5 $\mu$ g of DNA from each sample using the NEBnext DNA sample prep kit and were sequenced on a Genome Analyzer IIx in a 120-base-paired-end multiplex run. Sequencing yielded sets of $62,180,598-103,715,676$ total reads (table 1 ).

The four read sets were assembled to contiguous sequences (contigs) with the CLC Genomics Workbench V5 (CLC bio). Standard parameters for read trimming and de novo assembly were used applying an increased similarity value of 0.9 and $80 \%$ read coverage to obtain draft genome sets of microorganisms present in the respective samples. This yielded total contig lengths between 64,959 and 92,642 $\mathrm{Mb}$ (table 1).

Prokaryotic open reading frames (ORFs) of the individual draft genomes were predicted with GLIMMER3 [43], yielding sets ranging from 68,887 to 97,197 ORFs. The ORFs of each of the four samples were compared with BLASTP [44] against the NRPROT protein database [45]. About $80 \%$ of the ORFs of each of the four samples resulted in hits in NRPROT (table 2).

The first listed protein hit obtained by BLASTP comparison of each predicted ORF against NRPROT was analyzed for taxonomical assignment with MEGAN [46]. 
Table 1. Results obtained by illumina sequencing and subsequent assembly with the CLC Genomics Workbench V5 (CLC bio)

\begin{tabular}{lrll}
\hline Sample & Total reads, $\mathrm{n}$ & $\begin{array}{l}\text { Total contig } \\
\text { length, } \mathrm{Mb}\end{array}$ & $\begin{array}{l}\text { Average (maximal) } \\
\text { contig length, bp }\end{array}$ \\
\hline D0 & $102,523,070$ & 64,959 & $3,390(305,461)$ \\
R1 & $103,715,676$ & 68,167 & $4,533(679,591)$ \\
R2 & $62,180,598$ & 92,642 & $5,095(678,885)$ \\
R3 & $101,830,352$ & 69,314 & $3,991(482,208)$ \\
\hline
\end{tabular}

LCA ('last common ancestor') parameters were adapted for deduced protein analysis by applying a minimal support of one, and deactivation of the low complexity filter. This approach only provides estimates of the abundance of individual microorganisms in the microbiome since those bacteria with larger numbers of genes are overrepresented. However, it provides an initial taxonomical assignment and a statement about the presence or absence of microorganisms of interest, which in this case is C. difficile.

Pie charts shown in figure 1a summarize the percentages of deduced proteins assigned to phyla (major lineages) of the Bacteria domain. Most proteins were consistently assigned to the Firmicutes phylum in all of the four samples (68-73\%), in accordance with previous studies of healthy intestinal microbiota $[41,47,48]$. Interestingly, we also observed some differences between donor and recipient. Proteins assigned to the Verrucomicrobia phylum, which has only been recently identified as constituent of the healthy intestinal microbiome [49], were detected in all three of the recipient's samples (3-4\% of the deduced protein pools), but were almost undetectable in the donor's sample (fig. 1b). This indicates an adaptation of the donor's intestinal microbiome in the recipient intestinal tract following FMT. The composition of the recipient's intestinal microbiome was stable over a time period of 1-6 months after FMT, with only minor fluctuations (fig. 1a).

The approach described here is suitable to detect intestinal microorganisms up to the species level. In this case, it is of particular interest to determine the presence or absence of $C$. difficile in the recipient's intestine after FMT, as its absence would indicate successful treatment. Taxonomical assignment by MEGAN yielded only two significant hits to the $C$. difficile species in the four samples, and only a minor proportion of the analyzed deduced proteins were assigned to a possible C. difficile-related origin (species belonging to the Peptostreptococcaceae family). To further exclude the presence of C. difficile,
Table 2. ORF prediction and BLASTP analysis of deduced protein sequences

\begin{tabular}{lll}
\hline Sample & $\begin{array}{l}\text { Predicted } \\
\text { ORFs }\end{array}$ & $\begin{array}{l}\text { BLASTP against NRPROT, } \\
\text { hits/no hits }\end{array}$ \\
\hline D0 & 68,887 & $54,673 / 14,214$ \\
R1 & 69,997 & $56,352 / 13,645$ \\
R2 & 97,197 & $77,714 / 19,483$ \\
R3 & 72,235 & $57,469 / 14,766$ \\
\hline
\end{tabular}

all ORFs that yielded hits to C. difficile in MEGAN were additionally inspected by BLASTX and BLASTN comparisons against NRPROT and GenBank, respectively. Those remaining ORFs yielding hits in this comparison were assigned to their respective contigs, which were additionally inspected by BLASTX and BLASTN in the NCBI database.

One contig (No. 3183, 7,820 bp) of the R2 data set encodes a sigma-54-dependent transcriptional regulator (best hit in NRPROT WP_009903347; e-value 1e-144, $38 \%$ identity), an amidohydrolase family protein (WP_003439599; e-value 0.0, 53\% identity) and a renal dipeptidase family protein (best hit in NRPROT WP_003426730; e-value 0.0, 70\% identity). The other contig was identified in the D0 data set (contig No. 16465, $1,063 \mathrm{bp}$ ), which encodes a putative signaling protein (WP_003420236) of C. difficile. These findings were interpreted as rare exceptions and their origin still remains unclear because of the single occurrence.

In addition to these analyses, all contigs of the four datasets were screened for $r R N A$ operons showing similarities to $C$. difficile strain 630 (reference genome acc. No. NC_009089, position 10812-15968) and manually inspected. No hints for such an assignment were obtained, supporting the absence or rare presence of $C$. difficile in all four samples.

In contrast to $16 \mathrm{~S}$ rRNA-based methods, the deep sequencing approach reported herein also allows the detection of viruses. Consequently, we could detect about $10(8-11)$ types of bacteriophages in each of the four samples (fig. 1c), which might have been present as integrated prophages or free virus particles. In contrast, we found no hits to any potentially pathogenic eukaryotic viruses. However, it should be noted that the approach relies on prokaryotic ORF prediction, and is thus biased towards prokaryotic virus (bacteriophage) detection. The number of proteins assigned to bacteriophages compared with bacterial species was surprisingly low, 


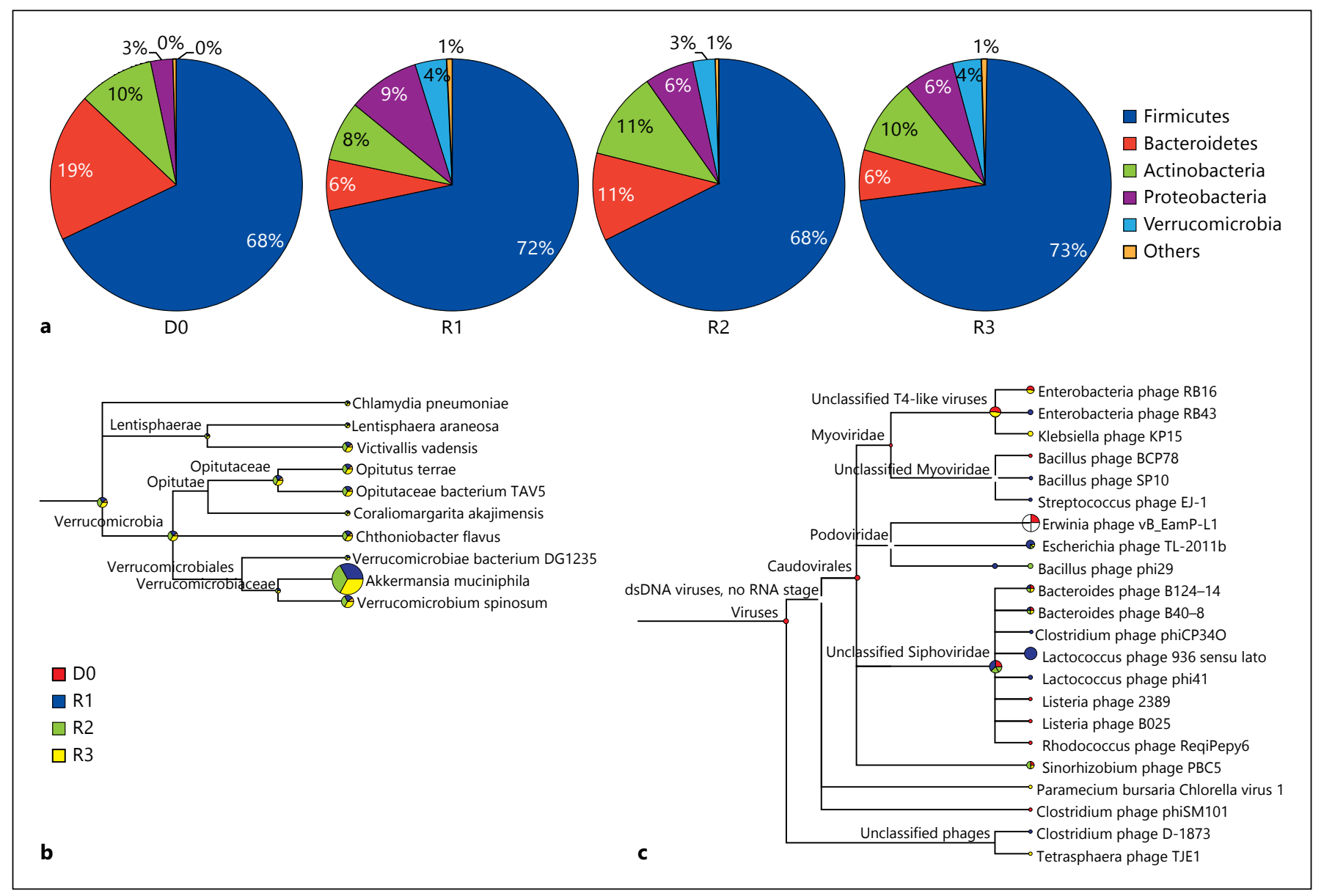

Fig. 1. Taxonomical assignment of proteins identified in intestinal microbiota with MEGAN. a Pie charts highlighting the BLASTP assignment of deduced proteins to bacterial phyla for the different samples. b Taxonomical assignment of the Verrucomicrobia phylum on the species level showing that these species were almost exclusively detected in the recipient's samples. c Detection of viruses. but is in accordance with reported findings [50]. The reasons for that remain unknown and will require further investigation.

\section{Discussion}

\section{Intestinal Microbiota and Microbiome}

In recent years, substantial progress has been made in understanding the composition of the gut microbiota due to the availability of affordable high-throughput sequencing techniques [51]. The discovery of numerous new bacterial species in the intestine [41] and the increased understanding of the complexity of the human microbiome have changed the conception of the human microbiome: the importance of the entire microbial population and its complex composition ('microbiota') in conjunction with the associated genes ('microbiome') is increasingly recognized as crucial for various aspects of health and disease. An estimated 100 trillion microorganisms $\left(10^{14}\right)$ are present in the human gastrointestinal tract [52], a quantity 10 times greater than the estimated number of all eukaryotic human cells per individual $\left(10^{13}\right)$. The microbiota coevolved with the host and supplies indispensable functions that humans cannot exert themselves, such as metabolic properties [53-56] or shaping of the adaptive and innate immune system [57-59].

\section{Fecal Transplantation for Recurrent CDAD}

Administering foreign stool in an attempt to manipulate the composition of the human intestinal microbiota is not a new concept. The first description of FMT dates back to 1958. Eiseman et al. [16] described the cases of 4 patients with pseudomembranous enterocolitis that were 
successfully treated with human enemas. Today, there are reports of more than 300 patients having undergone FMT for CDAD, mostly with impressive cure rates [14-37]. A systematic review of studies for FMT against CDAD published between 2000 and 2011 identified 124 patients of which $83 \%$ recovered immediately after the first FMT procedure [22]. Another recent systematic review found that of $273 \mathrm{CDAD}$ patients receiving FMT during the years 1946-2012, about 89\% experienced clinical resolution [32]. The successful FMT we report is in line with these previous reports.

\section{Deep Sequencing to Assess the Success of FMT}

We treated a patient suffering from recurrent CDAD with feces donated by the patient's sister after standard antibiotic therapy failed. The rationale for this approach has only recently been supplied when the human intestinal microbiome was first deciphered and shown to be of high complexity, with several hundred different bacterial species residing in the intestine of each individual [41, $60]$. This indicates that a healthy microbiota cannot easily be restored except by FMT from a healthy donor. This was largely unknown in most previous studies of FMT, but is now supported scientifically by increased understanding of the composition of the intestinal microbiota $[41,42,53,60]$.

We followed the FMT procedure by the only recently available metagenomic deep-sequencing technique, which provides a powerful tool to assess the microbial diversity residing in the human intestine $[41,42,53,60]$. Therefore, we employed the sequencing-by-synthesis approach to assess the intestinal microbial diversity of a patient with recurrent CDAD following FMT. We demonstrated that a complex and diverse microbiome was established in the patient employing a protocol that included de novo assembly of generated reads obtained from DNA of stool samples, prokaryotic ORF prediction, database alignment and taxonomical assignment. With this approach we could detect diverse and complex microbiomes predominated by Firmicutes in the donor as well as the recipient post-FMT. Furthermore, Verrucomicrobia species were identified only in the recipient's samples, reflecting the flexibility of the intestinal microbiota and an adaptation to the new host's intestinal microenvironment. Most importantly, no indications for a C. difficile infection were observed in any of the four samples, further indicating successful treatment of CDAD.

The sequencing approach we report is suitable to detect microorganisms up to the species level, which in contrast to $16 \mathrm{~S}$ rRNA-based methods is also suitable to iden- tify potentially pathogenic viruses. As reported herein, this approach may be used to exclude the presence of pathogens of interest, depending on the underlying disease and the patient's and donor's medical history.

One major drawback, however, is that only limited quantitative conclusions can be drawn, as the analysis is based on genes and therefore does not necessarily represent an abundance of microorganisms. It should be noted that the three samples from the recipient which were analyzed were obtained 6-7 months following FMT, and that unfortunately the recipient's stool sample at the time of FMT was unavailable due to inappropriate storage conditions. We therefore could not compare the microbial composition before and after FMT. However, the recipient's samples 6-7 months after successful FMT show a stable and diverse microbiome over a period of a month, predominated by Firmicutes, a hallmark of healthy intestinal microbiota, and no indication of $C$. difficile infection. Thus, the data indicates that a long-lasting reconstitution of the intestinal microbiome was achieved in the patient, accompanied with cessation of clinical symptoms of CDAD. At the time of writing, the patient has been free of symptoms for 3 years.

Recent studies applying 16S rRNA sequencing found that in CDAD patients who underwent successful FMT, a complex and diverse microbiome with highly abundant Firmicutes could be established [18, 21, 29]. In accordance with this, our study indicates that the donor's as well as the recipient's (post-FMT) intestinal microbiomes are mainly composed of Firmicutes (fig. 1a). In contrast, CDAD patients' (including those after unsuccessful FMT) microbiomes are predominated by Proteobacteria species [21], which have been detected in relatively low abundance in the samples analyzed herein (fig. 1a).

This is the first study to our knowledge to analyze intestinal microbiomes (metagenomes) of a patient with recurrent CDAD receiving FMT using the sequencing-bysynthesis approach and taxonomical classification based on coding sequences obtained from de novo genome assemblies to follow the course of treatment over time. In accordance with the previous reports, the healthy donor's as well as the recipient's (after successful FMT) intestinal microbiomes showed comparable richness and composition of healthy individuals. The patient's microbiome was of chimeric composition from donor and recipient.

Despite the high and still growing number of serious C. difficile infections worldwide [7,61], the number of patients subjected to FMT is still small. This may be due to patients' lack of knowledge and the fear of infections, as well as reluctance of physicians to a procedure that has 
barely been systematically evaluated and approved by medical authorities [62].

In the majority of published cases (around 80\%), FMT was performed during colonoscopy, but some delivery through a nasojejunal tube has been reported [33]. For reasons of simplicity and security, we applied the fecal suspensions through intra-anal application. The instillation of a high bacterial load influences CDAD-associated dysbiosis via competitive mechanisms, consequently leading to a sustained favorable restoration of the intestinal microbiota [34]. Dysbiosis, defined as an altered composition of intestinal bacterial species, is a precondition that allows pathogenic $C$. difficile to proliferate, ultimately causing overt infection [63]. A beneficial effect of FMT with a more limited number of microorganisms has been successfully shown in a small case series with a mixture of 10 facultative aerobic and anaerobic bacterial strains contained in saline solution: a mixture that comprised Enterococcus, Clostridium, Bacteroides spp. and E. coli [35].

A first culture-independent investigation of the microbial composition before and after FMT by means of terminal restriction fragment length polymorphism and sequencing of $16 \mathrm{~S}$ rRNA genes in one individual showed excellent concordance of microbial composition of the donor and recipient 14 days after FMT, whereas an abnormally low number of Firmicutes and Bacteroidetes was found in the gut microbiota of the recipient before transplantation [18]. We do not have any information about the abundance of Firmicutes in the recipient before FMT described here. The shift in intestinal microbial composition of a recipient induced by the transplantation procedure was shown to be sustained at least over a period of 24 weeks in a previous study [34]. We show that a healthy and diverse microbiome was established in our patient 6-7 months after FMT. The absence of CDAD symptoms in this patient for the last 3 years since the procedure suggests that a healthy intestinal microbiome has been maintained.

Concerns about transmission of bacterial, viral or parasitic pathogens, or even prions with donor stool, have been expressed. However, to the best of our knowledge, no case of such an infection in the course of FMT has been described to date. Approximately 3-15\% of all humans are asymptomatic carriers of $C$. difficile, with higher percentages in hospitalized patients [64]. Therefore, donor stool should be checked for the presence of $C$. difficile since transmission of spores or vital bacteria may hypothetically hamper successful restoration of the recipient's intestinal microbiota. Our analysis is suitable for that purpose, and in comparison to $16 \mathrm{~S}$ rRNA-based methods can identify viruses as well. However, the approach, as it relies on prokaryotic ORF prediction, is biased towards the identification of bacteriophages. Of note, bacteriophages have been shown to play important roles in the dynamics of the human intestinal microbiome in health and disease [65], and have been suggested to promote dysbiosis in inflammatory bowel disease [66]. Due to their potential roles in intestinal disease and their putative use as biomarkers, the need to include virome analyses in human microbiome projects has been expressed [67]. As conventional 16S rRNA-based metagenomic analyses cannot detect bacteriophages, alternative approaches, as the one reported here, are desired to include the virome, and could thus complement $16 \mathrm{~S}$ rRNA-based methods.

Additionally, the successful treatment of CDAD with FMT sheds light on other pathological conditions of the intestinal tract in which dysbiosis is considered a pathogenic factor. For instance, FMT has been successfully employed to treat cases of inflammatory bowel disease, irritable bowel syndrome and constipation [23, 68-70].

In conclusion, we have shown that a seriously ill patient can recover within a week after FMT from CDAD. The patient has been free of symptoms for a period of 3 years with a significant increase in quality of life. In addition, FMT is a fast, simple, well-tolerated and even cheap procedure.

\section{Acknowledgements}

The authors are grateful for the generous support of Prof. Martin Vingron and Prof. Hans Lehrach (Max Planck Institute for Molecular Genetics, Berlin), Prof. Dr. Erik C. Boettger (University of Zurich), and Prof. Peter H. Seeberger (Max Planck Institute of Colloids and Interfaces, Potsdam). We thank the patient for her cooperation, and Dr. Roberto F. Speck, MD, for his kind help with the manuscript. This work was supported by private financing (K.M.).

\section{Disclosure Statement}

The authors declare no competing interests.

References

\footnotetext{
1 Thomas C, Stevenson M, Riley TV: Antibiotics and hospital-acquired Clostridium difficile-associated diarrhoea: a systematic review. J Antimicrob Chemother 2003;51:1339-1350.

2 McDonald LC, Killgore GE, Thompson A, Owens RC Jr, Kazakova SV, Sambol SP, Johnson S, Gerding DN: An epidemic, toxin genevariant strain of Clostridium difficile. $\mathrm{N}$ Engl J Med 2005;353:2433-2441.
} 
3 Warny M, Pepin J, Fang A, Killgore G, Thompson A, Brazier J, Frost E, McDonald LC: Toxin production by an emerging strain of Clostridium difficile associated with outbreaks of severe disease in North America and Europe. Lancet 2005;366:1079-1084.

-4 Rouphael NG, O’Donnell JA, Bhatnagar J, Lewis F, Polgreen PM, Beekmann S, Guarner J, Killgore GE, Coffman B, Campbell J, Zaki SR, McDonald LC: Clostridium difficile-associated diarrhea: an emerging threat to pregnant women. Am J Obstet Gynecol 2008;198: 635.e1-e6.

$\checkmark 5$ Zilberberg MD, Shorr AF, Kollef MH: Increase in adult Clostridium difficile-related hospitalizations and case-fatality rate, United States, 2000-2005. Emerg Infect Dis 2008;14: 929-931.

-6 Nylund CM, Goudie A, Garza JM, Fairbrother G, Cohen MB: Clostridium difficile infection in hospitalized children in the United States. Arch Pediatr Adolesc Med 2011;165:451-457.

-7 Freeman J, Bauer MP, Baines SD, Corver J, Fawley WN, Goorhuis B, Kuijper EJ, Wilcox $\mathrm{MH}$ : The changing epidemiology of Clostridium difficile infections. Clin Microbiol Rev 2010;23:529-549.

8 Nelson R: Antibiotic treatment for Clostridium difficile-associated diarrhea in adults. Cochrane Database Syst Rev 2007;3:CD004610.

-9 Drekonja DM, Butler M, MacDonald R, Bliss D, Filice GA, Rector TS, Wilt TJ: Comparative effectiveness of Clostridium difficile treatments: a systematic review. Ann Intern Med 2011;155:839-847.

10 Cohen SH, Gerding DN, Johnson S, Kelly CP, Loo VG, McDonald LC, Pepin J, Wilcox MH, Society for Healthcare Epidemiology of America, Infectious Diseases Society of America: Clinical practice guidelines for Clostridium difficile infection in adults: 2010 update by the Society for Healthcare Epidemiology of America (SHEA) and the Infectious Diseases Society of America (IDSA). Infect Control Hosp Epidemiol 2010;31:431-455.

-11 Fekety R, McFarland LV, Surawicz CM, Greenberg RN, Elmer GW, Mulligan ME: Recurrent Clostridium difficile diarrhea: characteristics of and risk factors for patients enrolled in a prospective, randomized, double-blinded trial. Clin Infect Dis 1997;24:324-333.

-12 O’Neill GL, Beaman MH, Riley TV: Relapse versus reinfection with Clostridium difficile. Epidemiol Infect 1991;107:627-635.

$\checkmark 13$ Wilcox MH, Spencer RC: Clostridium difficile infection: responses, relapses and re-infections. J Hosp Infect 1992;22:85-92.

14 Bakken JS: Fecal bacteriotherapy for recurrent Clostridium difficile infection. Anaerobe 2009;15:285-289.

$\checkmark 15$ Bowden TA Jr, Mansberger AR Jr, Lykins LE: Pseudomembraneous enterocolitis: mechanism for restoring floral homeostasis. Am Surg 1981;47:178-183.

16 Eiseman B, Silen W, Bascom GS, Kauvar AJ: Fecal enema as an adjunct in the treatment of pseudomembranous enterocolitis. Surgery 1958;44:854-859.

17 Grehan MJ, Borody TJ, Leis SM, Campbell J, Mitchell H, Wettstein A: Durable alteration of the colonic microbiota by the administration of donor fecal flora. J Clin Gastroenterol 2010; 44:551-561.

18 Khoruts A, Dicksved J, Jansson JK, Sadowsky MJ: Changes in the composition of the human fecal microbiome after bacteriotherapy for recurrent Clostridium difficile-associated diarrhea. J Clin Gastroenterol 2010;44:354-360.

19 Schwan A, Sjölin S, Trottestam U, Aronsson B: Relapsing Clostridium difficile enterocolitis cured by rectal infusion of homologous faeces. Lancet 1983;ii:845.

20 Silverman MS, Davis I, Pillai DR: Success of self-administered home fecal transplantation for chronic Clostridium difficile infection. Clin Gastroenterol Hepatol 2010;8:471-473.

21 Shahinas D, Silverman M, Sittler T, Chiu C, Kim P, Allen-Vercoe E, Weese S, Wong A, Low DE, Pillai DR: Toward an understanding of changes in diversity associated with fecal microbiome transplantation based on $16 \mathrm{~S}$ rRNA gene deep sequencing. MBio 2012;3:e0033812.

22 Guo B, Harstall C, Louie T, Veldhuyzen van Zanten S, Dieleman LA: Systematic review: faecal transplantation for the treatment of Clostridium difficile-associated disease. Aliment Pharmacol Ther 2012;35:865-875.

23 Brandt LJ, Aroniadis OC: An overview of fecal microbiota transplantation: techniques, indications, and outcomes. Gastrointest Endosc 2013;78:240-249.

24 Rubin TA, Gessert CE, Aas J, Bakken JS: Fecal microbiome transplantation for recurrent Clostridium difficile infection: report on a case series. Anaerobe 2013;19:22-26.

25 Suwantarat N, Bobak DA: Fecal bacteriotherapy for recurrent Clostridium difficile infection: what's old is new again? Curr Infect Dis Rep 2013;15:101-103.

26 Rohlke F, Stollman N: Fecal microbiota transplantation in relapsing Clostridium difficile infection. Therap Adv Gastroenterol 2012;5: 403-420.

27 Brandt LJ: American Journal of Gastroenterology lecture: intestinal microbiota and the role of fecal microbiota transplant (FMT) in treatment of C. difficile infection. Am J Gastroenterol 2013;108:177-185.

28 Kleger A, Schnell J, Essig A, Wagner M, Bommer M, Seufferlein T, Härter G: Fecal transplant in refractory Clostridium difficile colitis. Dtsch Arztebl Int 2013;110:108-115.

29 Hamilton MJ, Weingarden AR, Unno T, Khoruts A, Sadowsky MJ: High-throughput DNA sequence analysis reveals stable engraftment of gut microbiota following transplantation of previously frozen fecal bacteria. Gut Microbes 2013;4:125-135.

30 Palmer R: Fecal matters. Nat Med 2011;17: 150-152.

-31 van Nood E, Speelman P, Kuijper EJ, Keller JJ: Struggling with recurrent Clostridium difficile infections: is donor faeces the solution? Euro Surveill 2009;14:pii $=19316$.

32 Kassam Z, Lee CH, Yuan Y, Hunt RH: Fecal microbiota transplantation for Clostridium difficile infection: systematic review and meta-analysis. Am J Gastroenterol 2013;108:500-508.

-33 Aas J, Gessert CE, Bakken JS: Recurrent Clostridium difficile colitis: case series involving 18 patients treated with donor stool administered via a nasogastric tube. Clin Infect Dis 2003;36:580-585.

34 Grehan MJ, Borody TJ, Leis SM, Campbell J, Mitchell H, Wettstein A: Durable alteration of the colonic microbiota by the administration of donor fecal flora. J Clin Gastroenterol 2010; 44:551-561.

35 Tvede M, Rask-Madsen J: Bacteriotherapy for chronic relapsing Clostridium difficile diarrhoea in six patients. Lancet 1989;1:1156-1160.

-36 Weingarden AR, Hamilton MJ, Sadowsky MJ, Khoruts A: Resolution of severe Clostridium difficile infection following sequential fecal microbiota transplantation. J Clin Gastroenterol 2013;47:735-737.

37 van Nood E, Vrieze A, Nieuwdorp M, Fuentes S, Zoetendal EG, de Vos WM, Visser CE, Kuijper EJ, Bartelsman JF, Tijssen JG, Speelman P, Dijkgraaf MG, Keller JJ: Duodenal infusion of donor feces for recurrent Clostridium difficile. N Engl J Med 201;368:407-415.

38 Hecht DW: Methods for Antimicrobial Susceptibility Testing of Anaerobic Bacteria, Approved Standard, ed 7. Wayne, Clinical and Laboratory Standards Institute, 2007.

39 Johnson S, Schriever C, Galang M, Kelly CP, Gerding DN: Interruption of recurrent Clostridium difficile-associated diarrhea episodes by serial therapy with vancomycin and rifaximin. Clin Infect Dis 2007;44:846-848.

40 Lowy I, Molrine DC, Leav BA, Blair BM, Baxter R, Gerding DN, Nichol G, Thomas WD Jr, Leney M, Sloan S, Hay CA, Ambrosino DM: Treatment with monoclonal antibodies against Clostridium difficile toxins. N Engl J Med 2010;362:197-205.

41 Qin J, Li R, Raes J, Arumugam M, Burgdorf KS, Manichanh C, Nielsen T, Pons N, Levenez F, Yamada T, Mende DR, Li J, Xu J, Li S, Li D, Cao J, Wang B, Liang $\mathrm{H}$, Zheng $\mathrm{H}$, Xie $\mathrm{Y}$, Tap J, Lepage P, Bertalan M, Batto JM, Hansen T, Le Paslier D, Linneberg A, Nielsen HB, Pelletier E, Renault P, Sicheritz-Ponten T, Turner K, Zhu H, Yu C, Li S, Jian M, Zhou Y, Li Y, Zhang X, Li S, Qin N, Yang H, Wang J, Brunak S, Doré J, Guarner F, Kristiansen K, Pedersen O, Parkhill J, Weissenbach J; MetaHIT Consortium, Bork P, Ehrlich SD, Wang J: A human gut microbial gene catalogue established by metagenomic sequencing. Nature 2010; 464:59-65.

42 Marchesi JR: Prokaryotic and eukaryotic diversity of the human gut. Adv Appl Microbiol 2010;72:43-62.

43 Salzberg SL, Delcher AL, Kasif S, White O: Microbial gene identification using interpolated Markov models. Nucleic Acids Res 1998;26:544-548. 
-44 Altschul SF, Gish W, Miller W, Myers EW, Lipman DJ: Basic local alignment search tool. J Mol Biol 1990;215:403-410.

-45 Benson DA, Karsch-Mizrachi I, Lipman DJ, Ostell J, Sayers EW: GenBank. Nucleic Acids Res 2009;37:D26-D31.

46 Huson DH, Auch AF, Qi J, Schuster SC: MEGAN analysis of metagenomic data. Genome Res 2007;17:377-386.

47 Wang X, Heazlewood SP, Krause DO, Florin TH: Molecular characterization of the microbial species that colonize human ileal and colonic mucosa by using $16 \mathrm{~S}$ rDNA sequence analysis. J Appl Microbiol 2003;95:508-520.

-48 Eckburg PB, Bik EM, Bernstein CN, Purdom E, Dethlefsen L, Sargent M, Gill SR, Nelson KE, Relman DA: Diversity of the human intestinal microbial flora. Science 2005;308 1635-1638.

49 van Passel MW, Kant R, Zoetendal EG, Plugge CM, Derrien M, Malfatti SA, Chain PS, Woyke T, Palva A, de Vos WM, Smidt H: The genome of Akkermansia muciniphila, a dedicated intestinal mucin degrader, and its use in exploring intestinal metagenomes. PLoS One 2011;6:e16876.

50 Arumugam M, Raes J, Pelletier E, Le Paslier D, Yamada T, Mende DR, Fernandes GR, Tap J, Bruls T, Batto JM, Bertalan M, Borruel N, Casellas F, Fernandez L, Gautier L, Hansen T, Hattori M, Hayashi T, Kleerebezem M, Kurokawa K, Leclerc M, Levenez F, Manichanh C, Nielsen HB, Nielsen T, Pons N, Poulain J, Qin J, Sicheritz-Ponten T, Tims S, Torrents D, Ugarte E, Zoetendal EG, Wang J, Guarner F, Pedersen O, de Vos WM, Brunak S, Doré J, MetaHIT Consortium, Antolín M, Artiguenave F, Blottiere HM, Almeida M, Brechot C, Cara C Chervaux C, Cultrone A, Delorme C, Denariaz G, Dervyn R, Foerstner KU, Friss C, van de Guchte M, Guedon E, Haimet F, Huber W, van Hylckama-Vlieg J, Jamet A, Juste C, Kaci G, Knol J, Lakhdari O, Layec S, Le Roux K, Maguin E, Mérieux A, Melo Minardi R, M’rini C, Muller J, Oozeer R, Parkhill J, Renault P, Rescigno M, Sanchez N, Sunagawa S, Torrejon A, Turner K, Vandemeulebrouck G, Varela E, Winogradsky Y, Zeller G, Weissenbach J, Ehrlich SD, Bork P: Enterotypes of the human gut microbiome. Nature 2011;473:174-180.
1 Sharon I, Morowitz MJ, Thomas BC, Costello EK, Relman DA, Banfield JF: Time series community genomics analysis reveals rapid shifts in bacterial species, strains, and phage during infant gut colonization. Genome Res 2013;23:111-120

52 Savage DC: Microbial ecology of the gastrointestinal tract. Annu Rev Microbiol 1977;31: 107-133.

53 Gill SR, Pop M, Deboy RT, Eckburg PB, Turnbaugh PJ, Samuel BS, Gordon JI, Relman DA, Fraser-Liggett CM, Nelson KE: Metagenomic analysis of the human distal gut microbiome. Science 2006;312:1355-1359.

54 Sonnenburg JL, Xu J, Leip DD, Chen $\mathrm{CH}$ Westover BP, Weatherford J, Buhler JD, Gordon JI: Glycan foraging in vivo by an intestine-adapted bacterial symbiont. Science 2005;307:1955-1959.

55 Grundmann O: The gut microbiome and presystemic metabolism: current state and evolving research. J Drug Metab Toxicol 2010;1: 105-111.

56 Ramotar K, Conly JM, Chubb H, Louie TJ: Production of menaquinones by intestinal anaerobes. J Infect Dis 1984;150:213-218.

57 Clavel T, Haller D: Bacteria- and host-derived mechanisms to control intestinal epithelial cell homeostasis: implications for chronic inflammation. Inflamm Bowel Dis 2007;13: 1153-1164.

58 Lee YK, Mazmanian SK: Has the microbiota played a critical role in the evolution of the adaptive immune system? Science 2010;330: 1768-1773.

-59 Mazmanian SK, Liu CH, Tzianabos AO, Kasper DL: An immunomodulatory molecule of symbiotic bacteria directs maturation of the host immune system. Cell 2005;122:107-118.

60 Ley RE, Peterson DA, Gordon JI: Ecological and evolutionary forces shaping microbial diversity in the human intestine. Cell 2006;124: 837-848.
61 Pearson A: Historical and changing epidemiology of healthcare-associated infections. J Hosp Infect 2009;73:296-304.

62 Kahn SA, Vachon A, Rodriquez D, Goeppinger SR, Surma B, Marks J, Rubin DT: Patient perceptions of fecal microbiota transplantation for ulcerative colitis. Inflamm Bowel Dis 2013;19:1506-1513.

63 Kyne L, Farrell RJ, Kelly CP: Clostridium difficile. Gastroenterol Clin North Am 2001;30: 753-777, ix-x.

64 Kato H, Kita H, Karasawa T, Maegawa T, Koino Y, Takakuwa H, Saikai T, Kobayashi K, Yamagishi T, Nakamura S: Colonisation and transmission of Clostridium difficile in healthy individuals examined by PCR ribotyping and pulsed-field gel electrophoresis. J Med Microbiol 2001;50:720-727.

65 Mills S, Shanahan F, Stanton C, Hill C, Coffey A, Ross RP: Movers and shakers: influence of bacteriophages in shaping the mammalian gut microbiota. Gut Microbes 2013; $4: 4-16$.

- 66 Lepage P, Colombet J, Marteau P, SimeNgando T, Doré J, Leclerc M: Dysbiosis in inflammatory bowel disease: a role for bacteriophages? Gut 2008;57:424-425.

67 Reyes A, Haynes M, Hanson N, Angly FE, Heath AC, Rohwer F, Gordon JI: Viruses in the faecal microbiota of monozygotic twins and their mothers. Nature 2010;466:334338.

68 Kunde S, Pham A, Bonczyk S, Teri C, Duba M, Conrad H Jr, Cloney D, Kugathasan S: Safety, tolerability, and clinical response after fecal transplantation in children and young adults with ulcerative colitis. J Pediatr Gastroenterol Nutr 2013;56:597-601.

-69 Damman CJ, Miller SI, Surawicz CM, Zisman TL: The microbiome and inflammatory bowel disease: is there a therapeutic role for fecal microbiota transplantation? Am J Gastroenterol 2012;107:1452-1459.

70 de Leon LM, Watson JB, Kelly CR: Transient flare of ulcerative colitis after fecal microbiota transplantation for recurrent Clostridium difficile infection. Clin Gastroenterol Hepatol 2013;11:1036-1038. 